\title{
Simulating Probabilistic Long-Term Effects in Models with Temporal Dependence
}

\author{
by Christopher Gandrud, Laron K. Williams
}

\begin{abstract}
The R package pltesim calculates and depicts probabilistic long-term effects in binary models with temporal dependence variables. The package performs two tasks. First, it calculates the change in the probability of the event occurring given a change in a theoretical variable. Second, it calculates the rolling difference in the future probability of the event for two scenarios: one where the event occurred at a given time and one where the event does not occur. The package is consistent with the recent movement to depict meaningful and easy-to-interpret quantities of interest with the requisite measures of uncertainty. It is the first to make it easy for researchers to interpret short- and long-term effects of explanatory variables in binary autoregressive models, which can have important implications for the correct interpretation of these models.
\end{abstract}

\section{Introduction}

Scholars from a wide variety of academic disciplines study phenomena with binary outcomes. This includes the study of war or peace (Beck et al., 1998), civil war or stability (Collier et al., 2003), wildlife habitat selection (Keating and Cherry, 2004), automobile accident severity (Al-Ghamdi, 2002), banking decisions (Maddala and Trost, 1982), labor force participation (Mroz, 1987), individual decisions about drinking water sources (Gelman et al., 2004), conflicts over water resources (Gleditsch et al., 2006), and education policy (Bailey et al., 2016), just to name a diverse few.

The desire to generalize produces the incentive for scholars to incorporate information both over time and across units, which results in time-series cross-sectional data. While helpful from an inferential standpoint, modeling processes that vary across time and space increase the number of potential estimation and interpretation problems facing scholars.

One problem that is unique to scholars examining binary time-series cross-sectional (BTSCS) data is the role of temporal dependence, or the notion that the probability of the occurrence of the event (i.e., the dependent variable) depends in part on how much time has passed since the previous occurrence. Whenever scholars estimate BTSCS models where there are omitted (or potentially unobservable) variables that are also correlated with time, there is a substantial risk of incorrect standard errors and highly misleading results (Beck et al., 1998).

Beck et al. $(1998,1261)$ offered a ground-breaking solution to this inferential obstacle by noting that "BTSCS data are grouped duration data", which implies that one can borrow techniques from duration analysis to properly model the influence of time since the previous event at some time $t$. This discovery led to a drastic increase in the number of scholars, political scientists in particular, employing duration modeling techniques with BTSCS data. Notable alternatives include dummy variables representing each value of $t$, splines, and cubic polynomials (Beck et al., 1998; Carter and Signorino, 2010). Put simply, these approaches assume that, for possibly un-modeled reasons, the probability of the event occurring at time $t$ is a function of how much time has elapsed since the event previously occurred.

At the same time, another movement has produced meaningful improvements in the interpretation of dynamic models in the social sciences. Over the last decade or so, scholars have improved our understanding of the various short- and long-term effects that arise from dynamic models. These longterm effects can take the form of long-range multipliers in autoregressive distributed lag models (De Boef and Keele, 2008) or dynamic simulations in models with lagged dependent variables (Williams and Whitten, 2012). In addition to providing a more complete picture of the inferences of key theoretical variables, one reason for the explosion in scholarly attention is the emphasis on providing appropriate measures of uncertainty with easy-to-implement software packages (e.g. Williams and Whitten, 2011; Gandrud et al., 2016; Choirat et al., 2017).

To this end, this article introduces the $R$ ( $R$ Core Team, 2017) package pltesim, which utilizes simulation methods to depict probabilistic long-term effects in binary models with temporal dependence (PLTE). The package is available from the Comprehensive R Archive Network (CRAN). The package follows the methodology introduced in Williams (2016). In the remainder of the paper, we will first discuss the methodological principles at work, then the process pltesim uses to calculate probabilistic long-term effects, and finally an example with various visualization approaches. 


\section{Long-term effects in models with temporal dependence}

Probabilistic long-term effects are the product of the intersection of two methodological trends: controlling for unmodeled duration dependence by including temporal dependence variables and interpreting short- and long-term effects of explanatory variables in autoregressive models. For example, if one is interested in the effects of $X$ on the probability of $Y$ at time $t$, and one controls for temporal dependence in any of the ways stated above, then $X$ will have both a short-term effect (interpreted in the traditional manner based on the link function) and a long-term effect. However, contrary to the calculation of long-term effects in dynamic models of continuous dependent variables (De Boef and Keele, 2008), the long-term effects in BTSCS models are probabilistic. As Williams (2016, 247) notes, "modifying the values of any of the independent variables at time $t$ potentially influences the predicted probabilities of the outcome in future time periods by forcing time since previous event to revert back to 0 , which itself affects the probability of observing the event".

Calculating probabilistic long-term effects involves a two-step process. The first step finds the change in the predicted probability of the outcome, given a change in the independent variable $\left(X_{K}\right)$, and a particular configuration of values of the other independent variables (or simulation scenario, $\mathbf{X}_{C}$ ). More formally, $\Delta \operatorname{Pr}\left(\hat{y}=1 \mid \mathbf{X}_{C}, \Delta X_{K}\right)$. A long-term effect occurs (by changing the values of the temporal dependence variables at future observations) if the observed outcome, $\hat{y}=1 \mid \mathbf{X}_{C}$ changes as a result of the change in $X_{K}$. The problem is that since this is a counterfactual, we never observe the actual outcome (just its probability). The change in the predicted probability of the event is typically the quantity of interest, and often is the point of emphasis when researchers interpret their results. In the calculation of PLTE, this quantity has a secondary interpretation as the likelihood of a variable having a PLTE. This is the change in the probability that $\hat{y}=1 \mid \mathbf{X}_{C}$, which also reflects the change in the probability that the time since previous event variables are reset to 0 at time $t+1 .^{1}$

The second step is to calculate the long-term effect (LTE). Assume that we have modeled temporal dependence in a simple fashion, with time representing a counter based on how many time periods have elapsed since the last event. The long-term effect, then, is the difference in the probability of the event occurring at time $t+1$ to $t+k$, given that an event occurred at time $t$, compared to the probability, given that the event did not occur at time $t$. Put another way, the LTE is a sequence of moving differences in the probability for two points along the hazard rate: one that assumes the event occurred at time $t$ and one that does not. If we use the notation that we establish above, we first set up a simulation scenario $\left(\mathbf{X}_{C}\right)$ containing the values of the independent variables (typically this would be the mean or median values) including time $(\bar{t})$. We then compare the probabilities of the event for this scenario-assuming that the time variable increases at each time period-to the scenario where the event occurred at time $t$ and the value of time resets to 0 at time $t+1$. The long-term effect at time $t+1$ is the following (L. K. Williams 2016, 248):

$$
\operatorname{LTE}_{\mathbf{X}_{\mathbf{C}}}^{t+1}=\operatorname{Pr}\left(\hat{y}=1 \mid \mathbf{X}_{\mathbf{C}} \text {, time }=0\right)-\operatorname{Pr}\left(\hat{y}=1 \mid \mathbf{X}_{\mathbf{C}} \text {, time }=\bar{t}\right) .
$$

Then the LTE is calculated at time $t+2$ by updating the values of time in both scenarios:

$$
\operatorname{LTE}_{\mathbf{X}_{\mathbf{C}}}^{t+2}=\operatorname{Pr}\left(\hat{y}=1 \mid \mathbf{X}_{\mathbf{C}} \text {, time }=1\right)-\operatorname{Pr}\left(\hat{y}=1 \mid \mathbf{X}_{\mathbf{C}} \text {, time }=\bar{t}+1\right) .
$$

And so on, up to a value of $k$, which represents the maximum or some other intuitive value of time. It is important to note that time-in addition to all the other temporal dependence variables derived from time such as splines or cubic polynomials-must be updated at each time period.

These probabilistic long-term effects can be modified so that they reflect a wide variety of quantities of interest. For example, scholars can easily depict the PLTE of a short-term change in $X_{K}$ (such as a one-unit change) or more lasting or permanent shocks in $X_{K}$. Figures can also depict the possibility of compounded effects, or the fact that having an event occur in one counterfactual increases the probability of future events. Finally, PLTE can provide interesting illustrations of the lasting effects of $X_{K}$ in models that explicitly model non-proportional hazards, such as when time is interacted with $X_{K}$. In the next section we provide an overview of the process of estimating PLTE using pltesim.

\section{pltesim Process}

pltesim is the only tool we know of that makes it easy to calculate and visualize probabilistic long-term effects in binary models with temporal dependence. pltesim has four steps:

\footnotetext{
${ }^{1}$ Two other considerations are important here. First, since these quantities are all based on estimates, then scholars interested in hypothesis testing must use the appropriate measures of uncertainty. Second, the quality of these quantities depends on the model's fit, so the interests of transparency requires that scholars provide measures of model fit.
} 
1. Find the parameter estimates. Currently pltesim works with binary outcome models, e.g. logit. So use a binary response with the glm function included with the default $\mathrm{R}$ installation.

2. Create a counterfactual scenario in a data. frame class object. This should have a row with the fitted counterfactual values and columns with names matching variables in your fitted model. All variables without values will be treated as having values of 0 in the counterfactual.

3. Simulate the long-term effects with pltesim's plte_builder function.

4. Plot the results with pltesim's plte_plot function.

In the next section we use simulated data from Williams (2016) to illustrate these steps.

\section{Examples}

The following examples replicate panels from Figure 1 in Williams $(2016,249)$. We start by loading the necessary packages:

library (pltesim)

library (ggplot2)

Notice that ggplot2 is loaded. It will be used later in this section to customize the plots created by pltesim.

The simulated data we will use in these examples is packaged with pltesim. It is called negative_year. The name refers to the simulated data having negative duration dependence. It has the following form:

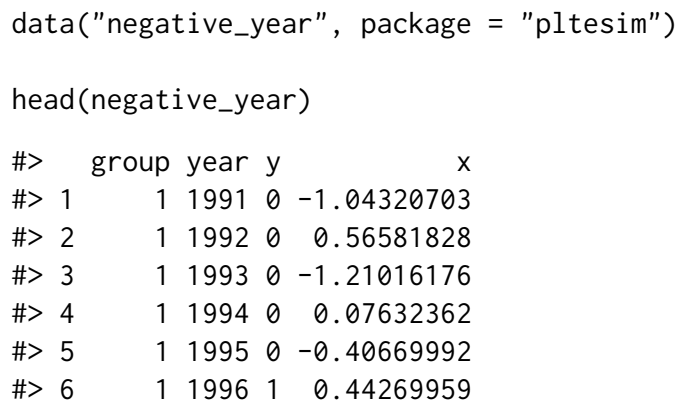

where $y$ is the binary response, $x$ is the non-time independent variable, year is the time variable, and group identifies each section of the panel.

Before finding the parameter estimates from this data, we need to create a standardized time variable that is in terms of time periods from the last spell (or the beginning of the observation period if left-censored), rather than years. pltesim includes the btscs function to accomplish this: ${ }^{2}$

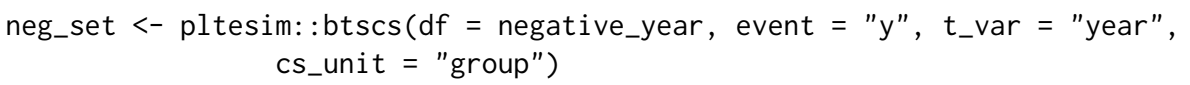

where $\mathrm{df}$ specifies the data frame. event is the binary response variable where 1 indicates an event, $\theta$ otherwise. $t_{\text {_nar }}$ specifies the time variable, cs_unit specifies the cross-sectional unit. The resulting data frame has the form:

\begin{tabular}{|c|c|c|c|}
\hline \#> & group year & $y$ & spell_time \\
\hline$\#>1$ & 11991 & $0-1.04320703$ & 1 \\
\hline$\#>2$ & 11992 & $0 \quad 0.56581828$ & 2 \\
\hline$\#>3$ & 11993 & $0-1.21016176$ & 3 \\
\hline$\#>4$ & 11994 & $0 \quad 0.07632362$ & 4 \\
\hline$\#>5$ & 11995 & $0-0.40669992$ & 5 \\
\hline \#> 6 & 11996 & 10.44269959 & 6 \\
\hline$\#>7$ & 21991 & $0-1.25522659$ & 1 \\
\hline$\#>8$ & 21992 & $0 \quad 0.29738988$ & 2 \\
\hline$\#>9$ & 21993 & $0 \quad 1.00741250$ & 3 \\
\hline 10 & 21994 & $0-0.42211204$ & 4 \\
\hline
\end{tabular}

${ }^{2}$ btscs is based on a function by the same name from the R package DAMisc which itself is based on the Stata (StataCorp, 2009) command implementing the procedure in Beck et al. (1998). btscs was included in pltesim to (a) allow improvements for handling single period spells, (b) match pltesim's syntax for ease of use within one workflow, and (c) to reduce pltesim's dependencies. It also starts the spell time counter at 1 rather than 0. 
This is the same data frame as before with the addition of a spell_time column containing a counter of time periods within each spell.

Now estimate the parameters:

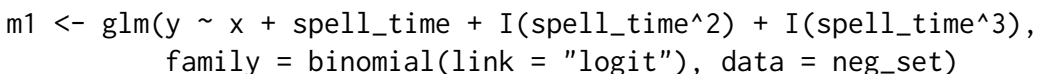

Note the inclusion of the I interpretation function to create the squared and cubed versions of spell_time. ${ }^{3}$ Additionally, the bs function from the splines package, included with R, allows similar inclusion of polynomial splines for time using the B-spline basis.

The change in $x$ is specified with:

$x_{-}$change $<-$data.frame $(x=0.5)$

which can be passed to plte_builder along with the fitted model object (m1). The counterfactual must be in the form of a data frame with column names matching each variable in the model and one row of fitted values. Variables from the model not included in the fitted value data frame will be treated as 0 .

The counterfactual is passed to plte_builder with the cf argument. The fitted model object is specified with obj. The time variable is identified with obj_tvar. Information about how long the change in $x$ persists in the simulation is given with cf_duration. It is permanent by default. The time period from the last spell over which to simulate the effects is given with the t_points argument.

The first simulation example finds the estimated impact of the counterfactual lasting for one time period. To do this, the plte_builder function's cf_duration argument is set to "one-time".

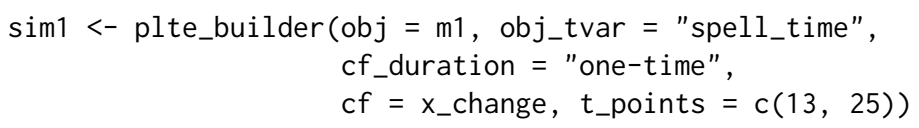

Running this code simulates a one period increase in $\mathrm{x}$ by 0.5 that occurs at 13 time points from the last spell. By default the central 95 percent interval of 1,000 simulations is returned. The extent of the returned central interval can be specified with plte_builder's ci argument and the number of simulations can be adjusted with the nsim argument.

We can now plot the results with the plte_plot function:

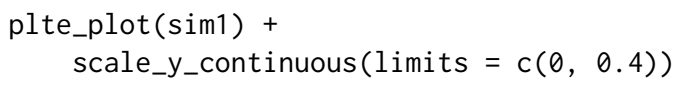

The first dot from the left in Figure 1 (and vertical dashed line) represents the median simulated baseline probability (and central 95 percent simulation interval) of the event occurring given the simulation scenario $\operatorname{Pr}\left(\hat{y}=1 \mid \mathbf{X}_{C}\right)$. The second dot from the left represents the updated probability of the event occurring given a one-time change in the variable of interest (or $\operatorname{Pr}\left(\hat{y}=1 \mid \mathbf{X}_{C}, \Delta X_{K}\right)$ ). In this case, the probability of the event occurring at time $t$ given $x=0.5$ is about 0.09 . The number labels next to the dots represent the values of $t$ in both scenarios.

One can assess whether the change in $X_{K}$ produces a statistically significant change in the probability of an LTE by determining whether the confidence intervals overlap. In this case, the increase in $X_{K}$ does not produce a statistically significant change in the probability for that time period. The remainder of Figure 1, however, reveals that the change in $X_{K}$ has a meaningful impact on the probability in future periods by changing the probability that the $t$ variable resets to 0 . The dashed lines from $t+1$ onwards are the 95 percent central simulation intervals for the probability of the event, given that the event did not occur at time $t: \operatorname{Pr}\left(\hat{y}=1 \mid \mathbf{X}_{C}\right.$, time $\left.=14 \ldots 25\right)$. The solid lines represent the counterfactual where $\hat{y}_{t}=1$. The two vertical lines at time $t+1$ illustrate how the value of $t$ either resets to 0 (if $Y_{t}=1$ ) or continues beyond its current value (if $Y_{t}=0$ ). Of the two scenarios, the counterfactual where the event does not occur $\left(Y_{t}=0\right)$ is much more likely given its small probability (0.09). The difference between these two vertical lines is the visual representation of the LTE from equation 1 . The intervals show that there is a statistically significant LTE from $t+1$ until $t+9$, at which point the central intervals overlap and there is no statistical difference between the two probabilities.

Note that because the output of plte_plot is a gg class ggplot2 object, we can modify it using the full set of ggplot2 functions, including in this case, the plot's y-axis limits with scale_y_continuous. This modification makes the plot more easily comparable with the ones that follow in this section.

To examine the effects of changes to $X_{K}$ that last for the entire simulation period, we set cf_duration $=$ "permanent". The results are show in Figure 2.

\footnotetext{
${ }^{3}$ This will allow plte_builder to identify the polynomials given just the base spell_time variable name.
} 


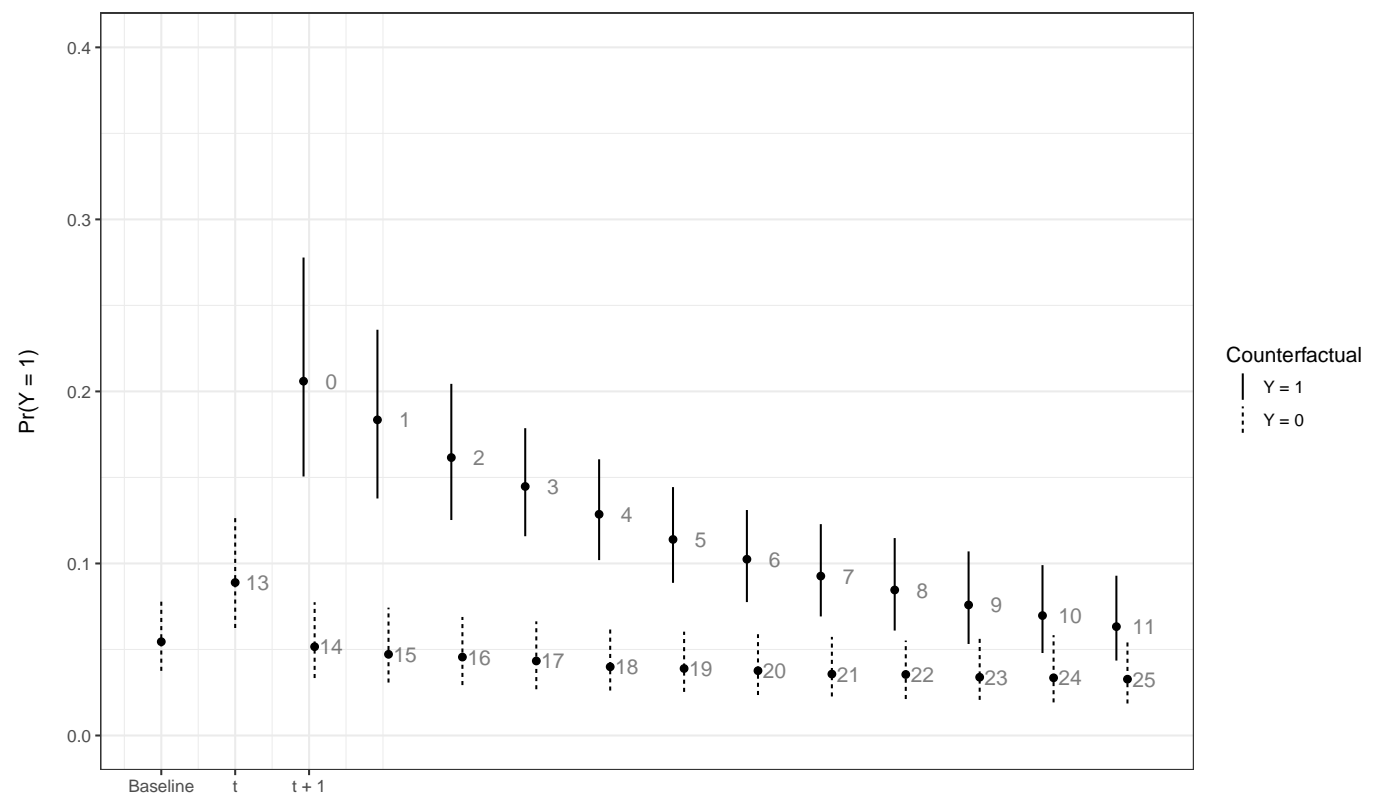

Figure 1: Simulated LTE with a one-period change in $\mathrm{x}$ by 0.5 .

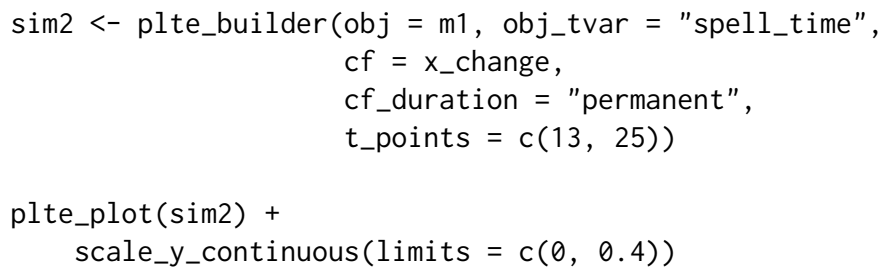

Users can also specify changes that last for periods shorter than the entire simulation period, but longer than one-period by supplying a numeric value to cf_duration. For example, to have the 0.5 increase in $x$ last for 4 time periods use:

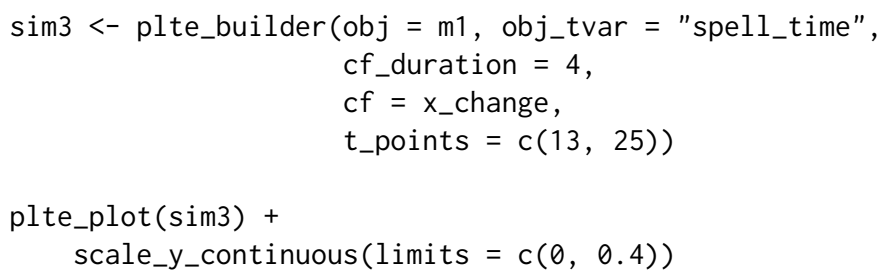

The results are shown in Figure 3.

Finally, we can use pltesim to examine not only the effects of changes in $x$, but also the compound effect of experiencing multiple events. To specify multiple events, supply an additional value to t_points. For example, to simulate and visualize the compound effect of an event at simulated time 20 use:

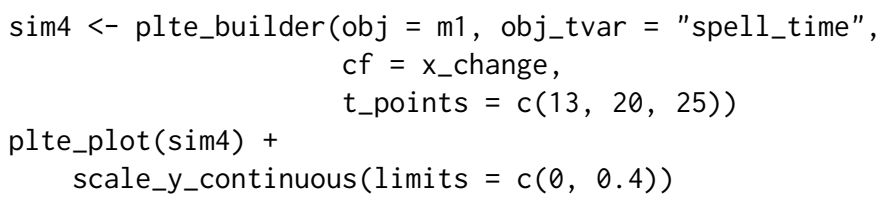

We can see in Figure 4 that in addition to the LTE given the event at time $t$ (with probability 0.09), there is a compounding effect that results in an even larger LTE because of the event at time $t+6$ (with probability of approximately 0.20$)$.

\section{Conclusion}

The goal of pltesim is to allow researchers to easily explore and present the short- and long-term effects of models estimated with temporal dependence. These variables can have a massive influence on the 


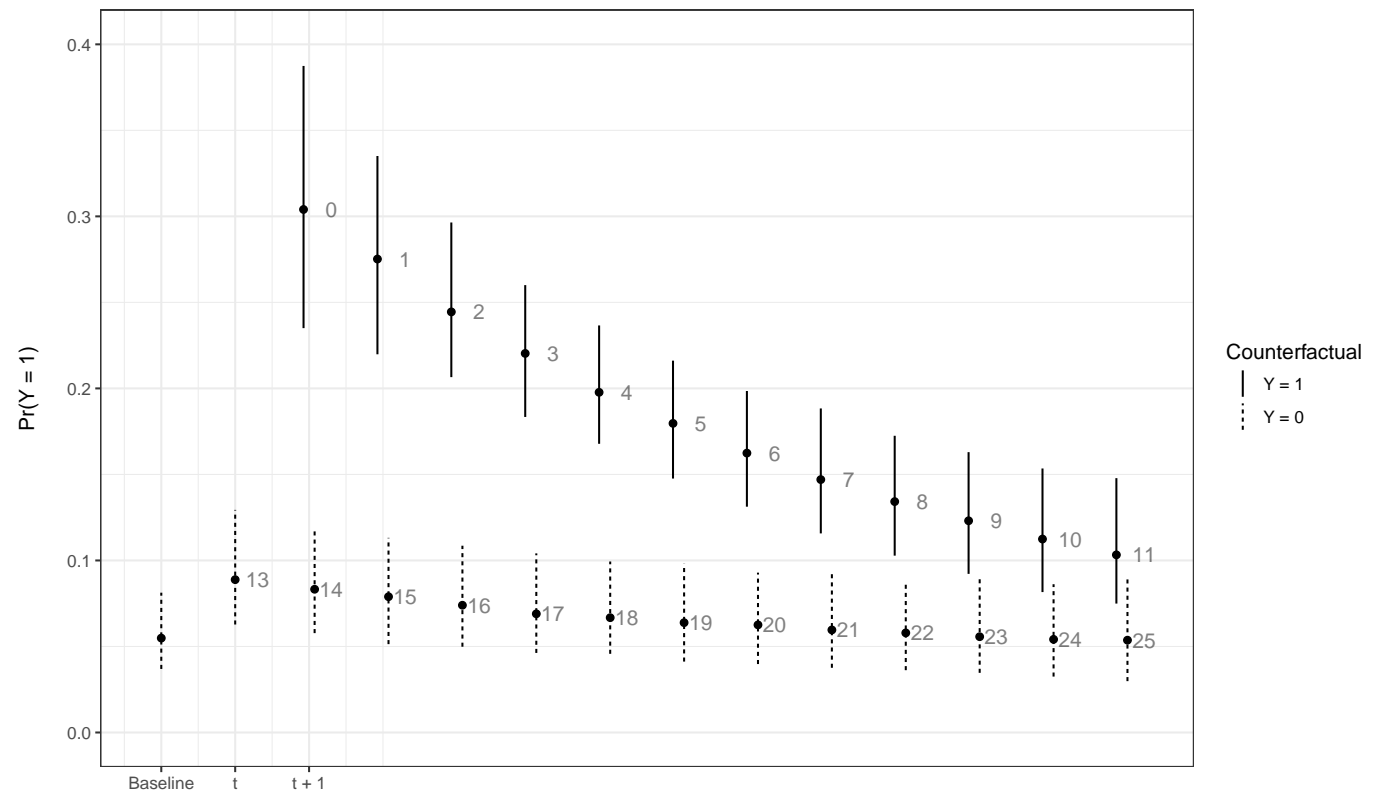

Figure 2: Simulated LTE with a permanent change in $x$ to 0.5 .

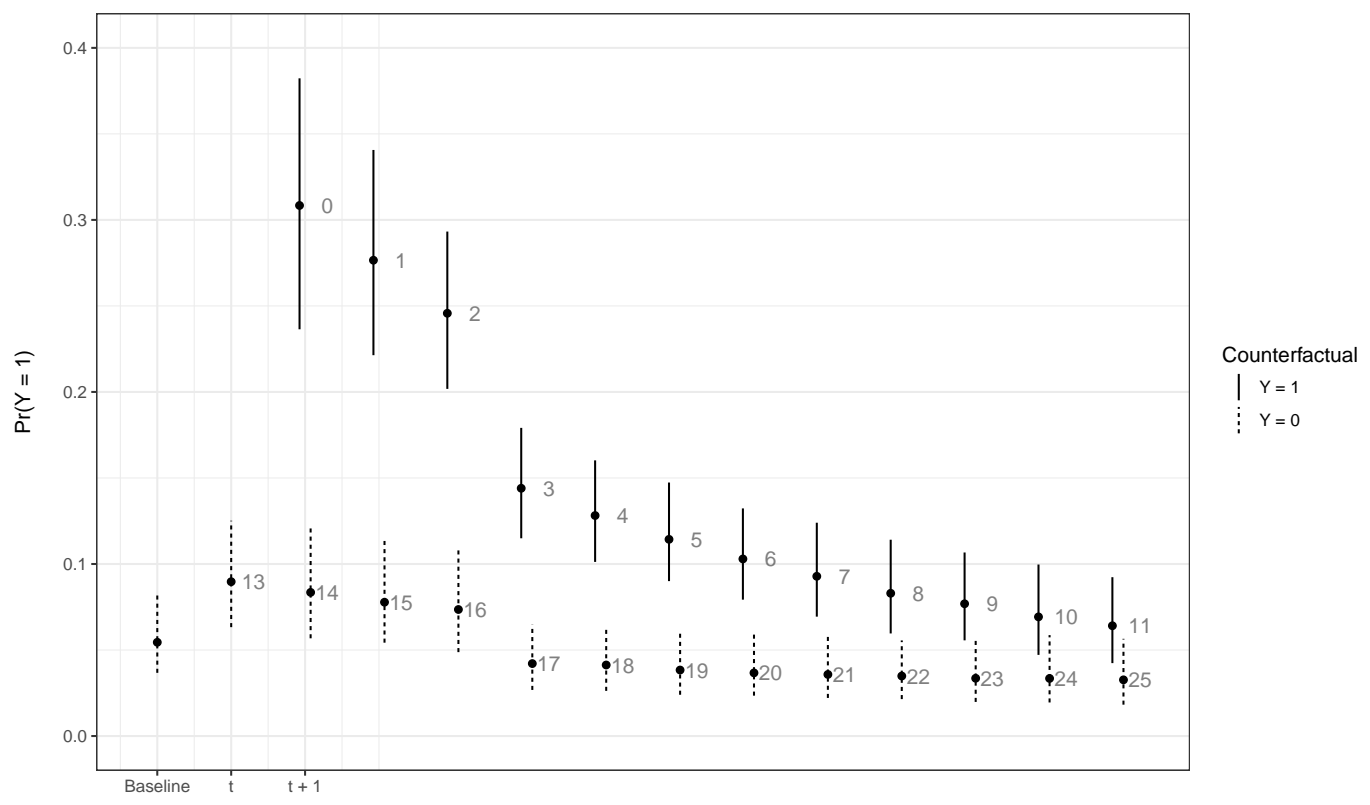

Figure 3: Simulated LTE with a four period increases of $x$ by 0.5 . 


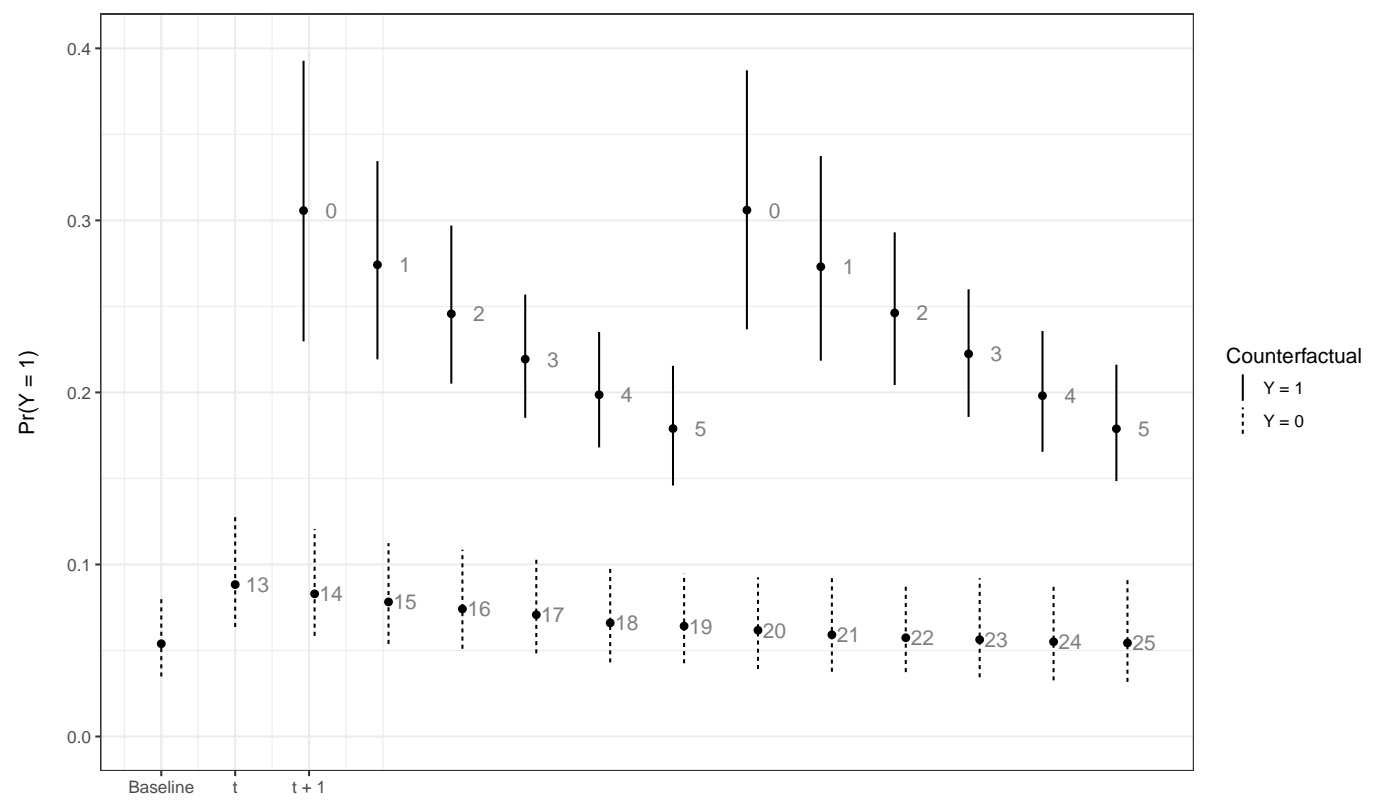

Figure 4: Simulated LTE with multiple events and a permanent increases of $x$ by 0.5.

outcome of interest and can change the substantive effects of key theoretical variables. Prominent theories (such as the conflict trap; see Collier et al., 2003) often have expectations that their variables have long-lasting effects, or that a variable's influence grows with each recurring event. Yet, up until the introduction of pltesim, scholars have been unable to estimate and graphically depict these theoretically interesting long-run dynamics from BTSCS models.

\section{Bibliography}

A. Al-Ghamdi. Using logistic regression to estimate the influence of accident factors on accident severity. Accident Analysis and Prevention, 34(6):729-741, 2002. [p401]

M. A. Bailey, J. S. Rosenthal, and A. H. Yoon. Grades and incentives: Assessing competing grade point average measures and postgraduate outcomes. Studies in Higher Education, 41(9):1548-1562, 2016. [p401]

N. Beck, J. Katz, and R. Tucker. Taking time seriously: Time-series-cross-section analysis with a binary dependent variable. American Journal of Political Science, 42(4):1260-1288, 1998. [p401, 403]

D. B. Carter and C. S. Signorino. Back to the future: Modeling time dependence in binary data. Political Analysis, 18:271-292, 2010. [p401]

C. Choirat, C. Gandrud, J. Honaker, K. Imai, G. King, and O. Lau. Zelig: Everyone's Statistical Software, 2017. URL https://cran.r-project.org/package=Zelig. R package version 5.0-18. [p401]

P. Collier, V. L. Elliot, H. Hegre, A. Hoeffler, M. Reynal-Querol, and N. Sambanis. Breaking the Conflict Trap. Oxford University Press, New York, 2003. [p401, 407]

S. De Boef and L. Keele. Taking time seriously. American Journal of Political Science, 52(1):184-200, 2008. [p401, 402]

C. Gandrud, L. K. Williams, and G. D. Whitten. Visualize dynamic simulations of autoregressive relationships in r. The Political Methodologist, 23(2):6-10, 2016. [p401]

A. Gelman, M. Trevisani, H. Lu, and A. Van Geen. Direct data manipulation for local decision analysis as applied to the problem of arsenic in drinking water from tube wells in bangladesh. Risk Analysis, 24(6):1597-1612, 2004. [p401]

N. P. Gleditsch, K. Furlong, H. Hegre, B. Lacina, and T. Owen. Conflicts over shared rivers: Resource scarcity or fuzzy boundaries? Political Geography, 25(4):361-382, 2006. [p401] 
K. A. Keating and S. Cherry. Use and interpretation of logistic regression in habitat-selection studies. Journal of Wildlife Management, 68(4):774-789, 2004. [p401]

G. S. Maddala and R. P. Trost. On measuring discrimination in loan markets. Housing Finance Review, 1 (3):245-268, 1982. [p401]

T. A. Mroz. The sensitivity of an empirical model of married women's hours of work to economic and statistical assumptions. Econometrica: Journal of the Econometric Society, 55(4):765-799, 1987. [p401]

R Core Team. R: A Language and Environment for Statistical Computing. R Foundation for Statistical Computing, Vienna, Austria, 2017. URL https://www.R-project.org/. Version 3.4.0. [p401]

StataCorp. Stata Statistical Software: Release 11. College Station, 2009. URL http://www. stata.com/. [p403]

L. K. Williams. Long-term effects in models with temporal dependence. Political Analysis, 24:243-262, 2016. [p401, 402, 403]

L. K. Williams and G. D. Whitten. Dynamic Simulations of Autoregressive Relationships. The Stata Journal, 11(4):577-588, 2011. [p401]

L. K. Williams and G. D. Whitten. But Wait, There's More! Maximizing Substantive Inferences from TSCS Models. Journal of Politics, 74(03):685-693, 2012. [p401]

Christopher Gandrud

Harvard University \& Zalando SE

Institute for Quantitative Social Science 1737 Cambridge Street Cambridge, MA, 02138, USA

ORCID: 0000-0003-4723-7585

christopher. gandrud@gmail.com

Laron K. Williams

University of Missouri

williamslaro@missouri.edu 